\title{
Surface Ionization State and Nanoscale Chemical Composition of UV- Irradiated Poly(dimethylsiloxane) Probed by Chemical Force Microscopy, Force Titration and Electrokinetic Measurements
}

Jing Song ${ }^{1}$, Jérôme F.L. Duval ${ }^{2}$, Martien A. Cohen Stuart ${ }^{3}$, Henrik Hillborg $^{1,4}$, Ullrich Gunst $^{5}$, Heinrich F. Arlinghaus ${ }^{5}$, G. Julius Vancso $^{1} *$

${ }^{1}$ University of Twente, Dutch Polymer Institute and MESA ${ }^{+}$Institute for

Nanotechnology, Materials Science and Technology of Polymers, P.O. Box 217, 7500 AE Enschede, The Netherlands

${ }^{2}$ Laboratory Environment and Mineral Processing, Nancy-University, CNRS, Research Center Francois Fiessinger, 15 Avenue du Charmois, BP40, 54501 Vandoeuvre-lèsNancy cedex, France

${ }^{3}$ Laboratory of Physical Chemistry and Colloid Science, Wageningen University P.O. Box 8038, 6700 EK Wageningen, The Netherlands

${ }^{4} A B B$ AB, Corporate Research, Power Technology, Västerås, SE 72178, Sweden ${ }^{5}$ Physikalisches Institut, Wilhelm-Klemm-Str. 10, Westfälische Wilhelms-Universität D48149 Münster, Germany

Email:j.song@tnw.utwente.nl,g.j.vancso@tnw.utwente.nl 


\section{Supporting information}

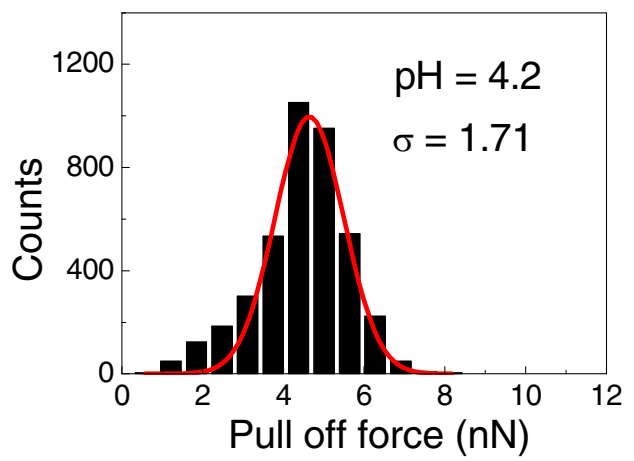

\section{Error! Bookmark not defined.}
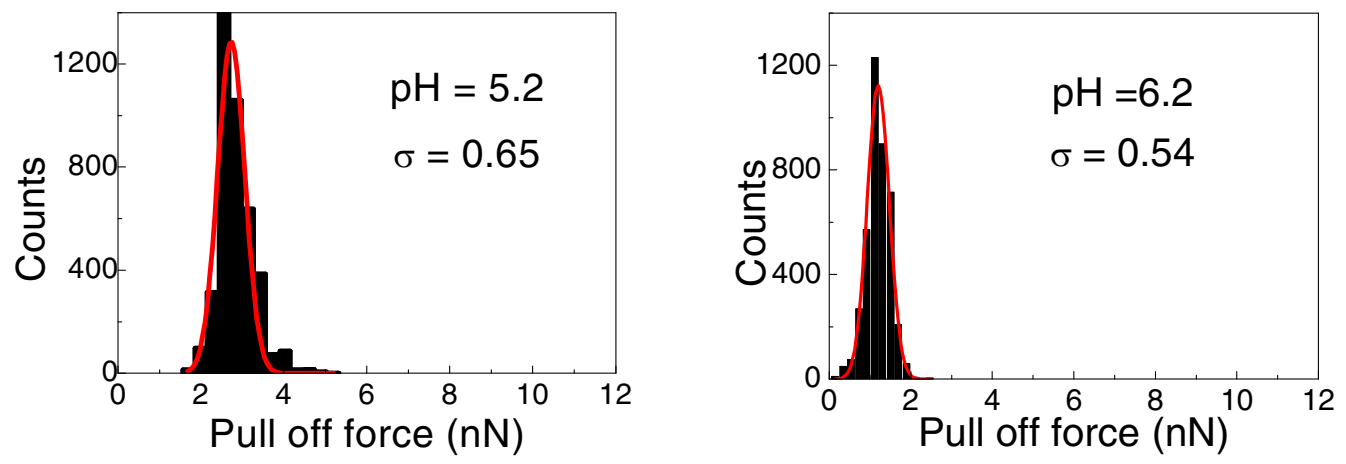

Figure S1. Representative histograms of the pull-off force recorded for PDMS exposed to $30 \mathrm{~min}$ UV/Ozone at different $\mathrm{pH}$. Scan size: $500 \mathrm{~nm} \times 500 \mathrm{~nm}$ (4096 force curves per histogram). The histograms were fitted using a Gaussian distribution $(\sigma=$ standard deviation).

As shown in Figure S1, it is clear that when using a hydroxyl functionalized tip, the average pull-off force decreases with increasing $\mathrm{pH}$. The histograms are fitted according to a Gaussian statistical distribution. The standard deviation of the Gaussian distribution $(\sigma)$ decreases from 1.93 to 0.54 with increasing $\mathrm{pH}$ reflecting a more homogenous distribution of pull off force. 


\section{Gouy-Stern Double Layer with Site Dissociation Model}

\section{Classical Gouy-Stern Double Layer}

Anisotropic ion accumulation exists at the contact interface between an electrochemically active surface and a liquid electrolyte. ${ }^{1}$ The charges at and adjacent to the surface will cause a potential difference between the region near the surface and the bulk of the solution. The potential decreases within the solution as a function of the distance from the charged surface. Specific ion adsorption occurrence is inferred from the dependence of certain double layer properties on the natures of counter and co-ions. Generally, ions that interact specifically (non-electro statically) with the surface approach it at a shorter distance.

The charge balance as a whole is written as

$$
\sigma^{0}+\sigma^{d}+\sigma^{a d s}=0
$$

$\sigma^{0}$ is the surface charge; $\sigma^{d}$ is the diffuse charge; $\sigma^{a d s}$ is the charge that stems from the adsorbed ions.

If $\sigma^{a d s}=0$, there is no specific ion adsorption.

The stern layer acts as a molecular condenser represented by a capacitance $C$. The surface potentials $\psi^{0}$, the potential at the oHp $\psi^{d}$ and the surface charges $\sigma^{d}$ are related via the capacitances. ${ }^{1}$

$$
C=\frac{\mathrm{d} \sigma^{d}}{\mathrm{~d}\left(\psi^{0}-\psi^{d}\right)}
$$

The plane where these specifically adsorbed ions reside is called the inner Helmholtz plane (iHp). The iHp layer capacitance and the oHp layer capacitances are given by 


$$
\begin{gathered}
C_{1}=\frac{\mathrm{d} \sigma^{0}}{\mathrm{~d}\left(\psi^{0}-\psi^{\beta}\right)} \\
C_{2}=\frac{\mathrm{d} \sigma^{d}}{\mathrm{~d}\left(\psi^{\beta}-\psi^{d}\right)}
\end{gathered}
$$

and

respectively.

$\psi^{\beta}$ is the potential at the inner Helmholtz plane. Within the framework of our analysis, the capacitance is considered constant (integral capacitances are then assimilated to differential capacitances), as commonly adopted in the literature. ${ }^{1}$

\section{Case Modeling: No Specific Ion-Adsorption}

For the description of charge and potential at the oxide-electrolyte interface, different models can be used. ${ }^{1-3}$ The surface charge at the oxidized PDMS-electrolyte interface originates from the dissociation of silanol groups when in contact with a $\mathrm{NaCl}$ buffer solution. The equilibrium constants of silanol ionization processes are $K_{\mathrm{a} 1}$ and $K_{\mathrm{a} 2}$ given by

$$
\begin{aligned}
& K_{a 1}=\frac{[-\mathrm{SiOH}]\left[\mathrm{H}^{+}\right] \exp \left(-e \psi^{0} / k T\right)}{\left[-\mathrm{SiOH}_{2}^{+}\right]} \\
& K_{a 2}=\frac{\left[-\mathrm{SiO}^{-}\right]\left[\mathrm{H}^{+}\right] \exp \left(-e \psi^{0} / k T\right)}{[-\mathrm{SiOH}]}
\end{aligned}
$$


where $\left[\mathrm{H}^{+}\right]$is the concentration of protons in the bulk and the other quantities in brackets are surface concentrations; $k$ is the Boltzmann factor; $T$ is the absolute temperature, $\psi^{0}$ is the surface potential and $e$ is the elementary charge.

Surface charge $\sigma_{0}$ derives from the acid-base interactions of the solution components and is defined by

$$
\sigma_{0}=e\left(\left[-\mathrm{SiOH}_{2}^{+}\right]-\left[-\mathrm{SiO}^{-}\right]\right)
$$

Consequently the $\mathrm{pH}$ of the solution is the primary externally adjustable variable, which together with the electrolyte concentration determines the sign and the magnitude of $\sigma_{0}$. In this study, neither formation nor disappearance of silanol groups is taken into account and the parameter $N s$ (effective number of ionizable surface sites) is therefore supposed to remain constant upon changing the electrolyte composition. The total amount of reactive silanol groups is directly dependent on the UV/Ozone treatment dose. We have

$$
\mathrm{Ns}=\left[-\mathrm{SiOH}_{2}^{+}\right]+\left[-\mathrm{SiO}^{-}\right]+[\mathrm{SiOH}]
$$

Diffuse ionic charge $\sigma^{d}$ can be derived from the Gouy-Chapman theory for a 1:1 electrolyte.

$$
\sigma^{d}=-\left(8 I \varepsilon_{0} \varepsilon_{r w} R T\right)^{1 / 2} \sinh \left(y^{d} / 2\right)
$$

where $\varepsilon_{0}$ is the dielectric permittivity of vacuum; $\varepsilon_{r w}$ is the relative permittivity of water; $I$ is the ionic strength of the solution; $R$ is the gas constant; $T$ is the temperature. $y^{d}$, the dimensionless potential defined by

$$
y^{d}=F \psi^{d} / R T
$$


where $\psi^{d}$ is generally assimilated to the electrokinetic potential $(\zeta)$.

The set of equations s5-s8 can be transformed into

$$
\sigma^{0}=e N_{s} \frac{\left(\left[H^{+}\right] / K_{a 1}\right) \exp \left(-e \psi^{0} / k T\right)-\left(K_{a 2} /\left[H^{+}\right]\right) \exp \left(e \psi^{0} / k T\right)}{1+\left(\left[H^{+}\right] / K_{a 1}\right) \exp \left(-e \psi^{0} / k T\right)+\left(K_{a 2} /\left[H^{+}\right]\right) \exp \left(e \psi^{0} / k T\right)}
$$

A consistent resolution -as achieved by numerical analysis- of equations s1, s2, s9, s11 and s12 allows for a quantitative determination of the double layer parameters that include the various potentials and charges entering the description of the interfacial structure. Further comparison with experimental data (i.e. electrokinetic potentials derived from streaming potential measurements) obtained for a broad range of ionic strengths and $\mathrm{pH}$ values enable the evaluation of the quantities $\mathrm{p} K \mathrm{a}_{1}$ and $\mathrm{pKa}$ and $N_{\mathrm{s}}$. For that purpose, a Least Square Regression (LSR) method was considered.

\section{Modeling In the Case Where There is Specific Ion-Adsorption}

Ions of the background electrolyte, which are not primarily charge-determining for a particular type of surface, may be specifically adsorbed onto the surface sites. The nonelectrostatic forces involved in this process result in shifts of the point of zero charge and the IEP. For such a reversible interface, the amount of specifically adsorbed ions is, for a given concentration and affinity of these ions, determined by the solution parameter $\mathrm{pH}$. In our experiment, the influence of specific ion adsorption is taken into account by formulating the appropriate equilibrium equations for phosphate groups.

In the low $\mathrm{pH}$ range, $\mathrm{SiOH}_{2}^{+}$are expected to be the predominant species present at the surface. The surface concentrations of $-\mathrm{SiOH}$ and $-\mathrm{SiO}^{-}$increase upon increasing the $\mathrm{pH}$ 
value. In the analysis, we considered adsorption of ions onto oppositely charged surface sites, which constitutes the most favorable situation from an energetic point of view. As revealed by electrokinetic experiments performed in $\mathrm{NaCl}$ solutions, $\mathrm{Na}^{+}$and $\mathrm{Cl}^{-}$may be considered as indifferent ions. Considering the neutral and negatively charged ions in the phosphate buffer solution, the adsorbability sequence for the various ions present in phosphate solutions onto oxide like surfaces is for low to intermediate $\mathrm{pH}$ values as follows $\mathrm{H}_{3} \mathrm{PO}_{4}\left\langle\left\langle\mathrm{H}_{2} \mathrm{PO}_{4}{ }^{-}\left\langle\left\langle\mathrm{HPO}_{4}{ }^{2-3}\right.\right.\right.\right.$. The binding of the pertaining anions onto PDMS surface are expressed by Equations 5-6 in section 3.7. The binding process equilibrium constants are $K_{\mathrm{x}}$ and $K_{\mathrm{y}}$. It is noted that the respective bulk concentrations of the various relevant phosphate ions were evaluated according to classical speciation calculations based on the first two dissociation $\mathrm{pKa}$ values reported in the literature. ${ }^{4-5}$ The charge density at the solid surface can be calculated from the total amount of charged surface groups that is now written:

$$
\begin{aligned}
N_{s} & =[\mathrm{SiOH}]+\left[\mathrm{SiOH}_{2}^{+}\right]+\left[\mathrm{SiO}^{-}\right]+\left[\mathrm{SiOH}_{4} \mathrm{PO}_{4}\right]+\left[-\mathrm{SiOH}_{2} \mathrm{PO}_{4}^{2-}\right] \\
\sigma^{0} & =\left[-\mathrm{SiOH}_{2}^{+}\right]-\left[\mathrm{SiO}^{-}\right]-2\left[-\mathrm{SiOH}_{2} \mathrm{PO}_{4}^{2-}\right]
\end{aligned}
$$

The charge density at the inner Helmholtz plane $\sigma^{\beta}$ is determined by the total concentration of adsorbed electrolyte ions:

$$
\sigma^{\beta}=\left[-\mathrm{SiOH}_{4} \mathrm{PO}_{4}\right]-2\left[-\mathrm{SiOH}_{2} \mathrm{PO}_{4}^{2-}\right]
$$

Due to the demand of charge neutrality in a stationary equilibrium, all charge densities must compensate each other as expressed by Equation (s1). From these basic equations 
the expressions for the concentrations of the different charged groups can be derived as a function of $N_{\mathrm{s}}, K_{\mathrm{a} 1}, K_{\mathrm{a} 2}, K_{\mathrm{x}}$ and $K_{\mathrm{y}}$. The parameters, $N_{\mathrm{s}}, K_{\mathrm{a} 1}$, and $K_{\mathrm{a} 2}$ were obtained from the analysis of the electrokinetic data obtained in $\mathrm{NaCl}$ indifferent electrolyte. On the basis of an iterative numerical analysis of equations s1, s2, s4-s6, s9, s11, s13-s15 and subsequent comparison (following a LSR strategy) with experimental data ( $\zeta$ potentials) at various ionic strengths and $\mathrm{pH}$ conditions, we could evaluate the searched quantities $K_{\mathrm{x}}$ and $K_{\mathrm{y}}$. We add that the values of the different electrical capacitances introduced in the model and chosen for the analysis were $\mathrm{C}=100 \mu \mathrm{F} \mathrm{cm} \mathrm{cm}^{-2}$ (used for the case where no specific adsorption takes place), $\mathrm{C} 1=120 \mu \mathrm{F} \mathrm{cm} \mathrm{cm}^{-2}$, and $\mathrm{C} 2=20 \mu \mathrm{F} \mathrm{cm}{ }^{-2}$ as used for the case of specifically adsorbing ions. ${ }^{3}$ The (assumed) constancy of these is certainly an abstraction from reality in view of the variation of the silanol distributions (or equivalently of the local balance between hydrophobic and hydrophilic silanol patches) with UV/Ozone treatment time. However, for the lack of better and for the sake of simplicity, we considered oxide-type capacitances as commonly reported in the literature. ${ }^{1,3}$ For that reason, the analysis of the data should certainly be considered at a semi-quantitative level but it has however the merit to reproduce the experimentally observed PDMS surface features, particularly the IEP shifts when varying the phosphate concentration in solution and/or the treatment time and the increase of the surface site concentration with increasing UV/Ozone time exposure. Those features are qualitatively in line with the independent AFM analysis.

\section{References}

1. Lyklema, J. Fundamentals of interface and colloid science Vol. II. Elsevier Academic Press, 2005. 
2. Duval, J.; Lyklema, J.; Kleijn J.M.; van Leeuwen, H.P. Langmuir 2001, 17, 7573.

3. Duval, J.; Kleijn, J.M.; Lyklema, J.; van Leeuwen, H.P. J. Electroanal. Chemistry 2002, 532, 337.

4. Ong, S.W.; Zhao, X.L.; Eisenthal, K.B. Chem. Phys. Lett. 1992, 191, 327.

5. Wang, B.; Abdulali-Kanji, Z.; Dodwell, E.; Horton, J.H.; Oleschuk, R.D. Electrophoresis 2003, 24, 1442. 